\title{
Fast spin echo sequences for BOLD functional MRI
}

\author{
Benedikt A Poser • David G Norris
}

Received: 28 June 2006 / Accepted: 25 October 2006 / Published online: 24 January 2007

(C) ESMRMB 2007

\begin{abstract}
At higher field strengths, spin echo (SE) functional MRI (fMRI) is an attractive alternative to gradient echo (GE) as the increased weighting towards the microvasculature results in intrinsically better localization of the BOLD signal. Images are free of signal voids but the commonly used echo planar imaging (EPI) sampling scheme causes geometric distortions, and $\mathrm{T}_{2}$ * effects often contribute considerably to the signal changes measured upon brain activation. Multiply refocused SE sequences such as fast spin echo (FSE) are essentially artifact free but their application to fast fMRI is usually hindered due to high energy deposition, and long sampling times. In the work presented here, a combination of parallel imaging and partial Fourier acquisition is used to shorten FSE acquisition times to near those of conventional SE-EPI, permitting sampling of eight slices (matrix $64 \times 64$ ) per second. Signal acquisition is preceded by a preparation experiment that aims at increasing the relative contribution of extravascular dynamic averaging to the BOLD signal. Comparisons are made with conventional SE-EPI using a visual stimulation paradigm. While the observed signal changes are approximately $30 \%$ lower, most likely due to the absence of $\mathrm{T}_{2} *$ contamination, activation size and t-scores are comparable for both methods, suggesting that HASTE fMRI is a viable alternative, particularly if distortion free images are required. Our data also indicate that the BOLD post-stimulus undershoot is most probably attributable to persistent elevated
\end{abstract}

B. A. Poser $(\varangle) \cdot$ D. G. Norris

FC Donders Centre for Cognitive Neuroimaging,

Trigon 181, P. O. Box 9101, 6500 HB Nijmegen,

The Netherlands

e-mail: benedikt.poser@fcdonders.ru.nl oxygen metabolism rather than to delayed vascular compliance.

Keywords BOLD fMRI - Fast spin echo - HASTE . parallel imaging · BOLD undershoot · Balloon model

\section{Introduction}

Echo planar imaging (EPI) [1] is commonly used for functional MRI (fMRI). It finds application in most methods for blood oxygenation level dependent (BOLD) fMRI [2] as well as other functional imaging sequences such as arterial spin labeling (ASL) [3] or vascular space occupancy (VASO) [4] fMRI. For BOLD fMRI, gradient echo (GE) EPI is popular as whole-brain images with high functional contrast to noise (CNR) can typically be acquired at a sufficiently high temporal resolution to adequately sample the BOLD response. An attractive alternative for fMRI at high field strengths is provided by spin echo (SE) based $\mathrm{T}_{2}$ contrast, which arises from changes in extravascular dynamic averaging and intravascular changes in the effective $\mathrm{T}_{2}$ of blood, both associated with the BOLD effect. Signal attenuation due to dynamic averaging is diffusion-induced and, in contrast to static dephasing effects in the field inhomogeneities around larger vessels, is not refocused in the spin echo experiment as it is a random process. At higher field strengths, this leads to an intrinsically better spatial specificity, as the relative contribution of the intravascular compartment is reduced, and the signal is weighted towards the microvasculature, that is expected to be closer to the 'true' site of neuronal activity [5-10]. While at $1.5 \mathrm{~T}$ the intravascular contribution to the SE fMRI signal dominates [11], the intravascular 
signal nearly vanishes at ultra-high field strength such as $9.4 \mathrm{~T}$ [7]. At $3 \mathrm{~T}$ still approximately half of the functional signal change is of intravascular origin $[12,13]$, but the increased specificity of SE over GE could be demonstrated [14]. Quantitative measurement of the spatial extent of the BOLD response [6] showed a $13 \%$ narrower point spread function for SE-EPI, albeit at the cost of a factor 3 reduction in functional CNR. An additional advantage of SE imaging is its insensitivity to through-plane susceptibility gradients which can result in substantial signal voids in GE images. It was shown in Ref. [12] that parts of the inferior prefrontal brain activation associated with cognitive tasks could be imaged with SE-EPI, but not GE-EPI. However, SE-EPI suffers from in-plane distortions, and some $\mathrm{T}_{2} *$ weighting will remain, caused by the long EPI readout of typically $40 \mathrm{~ms}[15,16]$. The severity of $\mathrm{T}_{2} *$ related artifacts increases rapidly with field strength due to the shortened relaxation time. As pointed out in Ref. [15] $\mathrm{T}_{2} *$ effects can further lead to a broadening of the PSF, causing the effective spatial resolution to be less than the voxel size in regions of short $\mathrm{T}_{2}{ }^{*}$, particularly at higher field strengths (4 and $7 \mathrm{~T})$ as used in their study.

BOLD fMRI with pure $T_{2}$ contrast, and with no EPI artifacts, can be performed by using a multiplyrefocused sequence, such as fast spin echo (FSE). This was demonstrated in an early study at $1.5 \mathrm{~T}$ [17] with a fully sampled multi-shot FSE sequence at relatively high in-plane resolution $\left(1.6 \times 3.2 \mathrm{~mm}^{2}\right)$. The resulting long repetition time (TR) of $>20 \mathrm{~s}$ for five slices, however, only allowed use of very long block stimuli. At the field strength of $1.5 \mathrm{~T}$ the FSE sequence was mainly sensitive to intravascular BOLD effects, but it was suggested in [17] that by adjusting the echo spacing the sensitivity could be tuned towards a specific range of vessel sizes. At $3 \mathrm{~T}$, the increased contribution of extravascular dynamic averaging effects can be utilized. If for a given TE the echo train for signal acquisition is preceded by a preparation period with a long refocusing interval, the relative contribution from the extravascular compartment, and thus the more desirable portion of the BOLD contrast can be increased. As in SE-EPI, the centre of $k$-space should be acquired at a TE approximately equal to $\mathrm{T}_{2}$. Sampling times can be made compatible with the requirements for fast fMRI by the use of partial Fourier and parallel imaging. By following this sampling strategy, repetition times comparable to that of SE-EPI can be realized (see Methods section). Acquisition rates of eight slices per second can be achieved, permitting good sampling of the BOLD response with near whole brain coverage in about $3 \mathrm{~s}$.

In this paper, the value of such an approach is investigated, and comparisons are made with the more conven- tional, singly refocused SE-EPI sequence (some authors also refer to the spin echo in this context as the Hahn spin echo). To facilitate a conservative comparison of functional sensitivity, full $k$-space acquisition is used for SE-EPI.

\section{Methods}

Data acquisition

Two practical problems must be overcome to make fast multi-slice experiments viable as an alternative to SE-EPI. First, image acquisition times should ideally not exceed those of a conventional SE-EPI experiment. Second, the energy deposition of the refocusing pulses must not exceed specific absorption rate (SAR) limits; yet a high enough signal to noise (SNR) must be achieved at sufficient spatial resolution. Both constraints may be addressed by using a combination of partial Fourier (PF) imaging, and parallel imaging such as SMASH [18], SENSE [19] or GRAPPA [20].

To reduce total acquisition time, we implemented the HASTE sequence (half-Fourier acquisition single shot turbo SE) with linear ascending phase-encoding sampling scheme with acceleration factor 4 and $7 / 8 \mathrm{PF}$. The first echo is refocused at $50 \mathrm{~ms}$ by a three-lobed $180^{\circ}$ selective sinc pulse. All following pulses are $2 \mathrm{~ms}$ single-lobed sinc pulses. Sampling starts on the partially acquired side of $k$-space. Echo spacing was adjusted such that the $k$-space centre is acquired at the 'target' TE of $80 \mathrm{~ms}$ (at $3 \mathrm{~T}$ ), giving an inter-echo TE of $6.5 \mathrm{~ms}$. Under the conservative assumption of a tissue $\mathrm{T}_{2}$ of $80 \mathrm{~ms}$ the line-broadening in this sequence caused by transverse relaxation is less than one pixel. The sequence is shown schematically in Fig. 1. Images were reconstructed offline in Matlab (The MathWorks, Inc., USA) using SENSE [19] reconstruction. Sensitivity maps were calculated from separate moderately smoothed (FWHM 3 pixels) full resolution FLASH scans, using a third order polynomial fit for extrapolation beyond the edge of the object. The acquisition parameters were as follows: matrix size $64 \times 64, \mathrm{FoV}=224 \mathrm{~mm}$, voxel size $3.5 \times 3.5$ $\times 5 \mathrm{~mm}^{3}, \mathrm{TR}=2 \mathrm{~s}$, pixel bandwidth $300 \mathrm{~Hz}$, effective echo train length of $14 k$-space lines and $T_{\text {acq }}=130 \mathrm{~ms}$ per slice. Five slices covering the visual cortex were acquired. Fully sampled SE- and GE-EPI measurements were made using a singly refocused double-echo sequence, with the same geometric parameters as for HASTE and at a bandwidth of $2,700 \mathrm{~Hz} /$ pixel. For the EPI sequence, this resulted in a total acquisition time $T_{\text {acq }}=120 \mathrm{~ms}$ per slice. Images were reconstructed online using the product image reconstruction. All data 
were acquired on a $3 \mathrm{~T}$ Siemens Magnetom Trio system (Siemens Medical Solutions, Erlangen, Germany) using the product eight-channel head coil.

Stimulus material and subjects

Visual stimuli consisting of $30 \mathrm{~s}$ rest followed by $21 \mathrm{~s}$ of $8 \mathrm{~Hz}$ inverting black and white checkerboards were shown using Presentation software (Neurobehavioral Systems, Inc., USA). Each experimental run lasted 6 min (180 images). Scan order was alternated between sessions. Six subjects with normal or corrected-to-normal vision were scanned, after having given written consent according to local regulations.

\section{Data processing and analysis}

Linear trend removal and high-pass filtering of all time courses were carried out in Brainvoyager 2000 (Brain Innovation, The Netherlands). $T$ tests were performed at $P<0.0001$ (all $p$ values uncorrected for small volumes). Mean and maximum $t$-scores, as well as count of active voxels were noted. Functional CNR was calculated for each subject as the average signal change divided by the mean error on the average stimulus response curve. In addition, a spatial map of pixel-wise temporal SNR (tSNR) was calculated in one dataset to assess the effect of parallel imaging on temporal stability.

\section{Results}

Stable SENSE reconstruction of the $T_{2}$ prepared HASTE images could be achieved despite the high acceleration factor. Only in some cases could weak residual fold-over be observed. Figure 2 shows GE-EPI, SE-EPI and HASTE images for comparison. Figure $3 a$ and $b$ show corresponding slices from SE-EPI and HASTE experiments in one functional subject. The activation overlay represents $t$-scores at $P<0.0001$. Temporal SNR of the two preprocessed time courses is shown in Fig. 3c and $\mathrm{d}$. In the occipital cortex, tSNR of HASTE is comparable to that of SE-EPI, but lower in other regions. The spatial noise variation not only shows the expected dependence on tissue type, but also clearly resembles that of the $g$-map (Fig. 3e). The $g$-factor is the coil geometry related noise increase caused by the use of parallel imaging [19]. Figure $3 f$ illustrates that the visual cortex is not strongly affected by $g$-noise; typical values here are between 2.0 and 2.6. Higher values of to up to 6 were observed in central brain regions.
The average $(n=6)$ BOLD response curves for SE-EPI and HASTE measurements are shown in Fig. 4 (top). The time evolution of both curves is identical, however, the maximum signal change in SE-EPI $(1.88 \%)$ is higher than in the HASTE measurement $(1.33 \%)$, at comparable baseline intensities. Both methods show that about $9 \mathrm{~s}$ is required for the signal to return to baseline, followed by a BOLD undershoot. Normalizing the activation time courses shows that both main response and undershoot of HASTE are proportionately reduced compared to SE-EPI, and the curves are almost perfectly aligned (not shown).

At $P<0.0001$, average activation volumes are comparable in SE-EPI (332 voxels) and HASTE (305 voxels). Averages of mean and maximum $t$-scores in the HASTE measurements are $7.25 \pm 0.37$ and $16.42 \pm 1.86$, respectively, and $7.35 \pm 0.32$ and $16.12 \pm 1.72$, respectively for SE-EPI. Considering only voxels active in both scans yields a similar picture, with $7.81 \pm 0.75$ and $16.30 \pm 2.04$ for HASTE, and $7.99 \pm 0.59$ and $16.01 \pm$ 1.87 for SE-EPI. Differences in $t$-scores are not significant (permutation test, $P<0.05$ ) and much smaller than the natural variation between subjects. Calculation of functional CNR yields 16.55 (noise 0.12 ) for SE-EPI, and 11.19 (noise 0.11) for HASTE; this means that the differences in sensitivity can be explained by the differences in signal change amplitude alone.

\section{Discussion}

Considering the acquisition parameters used and the $g$-factors observed in the occipital lobe, 'within-image' SNR of HASTE would be expected to be approximately $30 \%$ lower than that of SE-EPI. The observation of comparable effective temporal SNR of both methods (in the visual areas) is attributable to the contribution of physiological noise which markedly reduces the relative effect of image noise, and thus the use of parallel imaging on temporal stability [21]. In central brain areas, however, much higher $g$-factors and the reduced tSNR suggest that functional sensitivity would be compromised. The $g$-noise can be reduced by a choosing lower acceleration factor and/or different coil array, as the $g$-maps are uniquely related to the combination of coil geometries with acceleration factor $a$, and $g$-values rapidly grow with the number of pixels overlapping in the foldover (cf. Fig. 3). While a protocol with acceleration factor 3 might therefore produce a 'more benign' $g$-map, it would not have allowed meeting our demands on $T_{\text {acq }}$ and target $\mathrm{TE}$ of $80 \mathrm{~ms}$, and required use of ambitious $\mathrm{PF}(<60 \%)$ if the number of refocusing pulses were to be kept constant. On future systems this is will not be an issue as the 


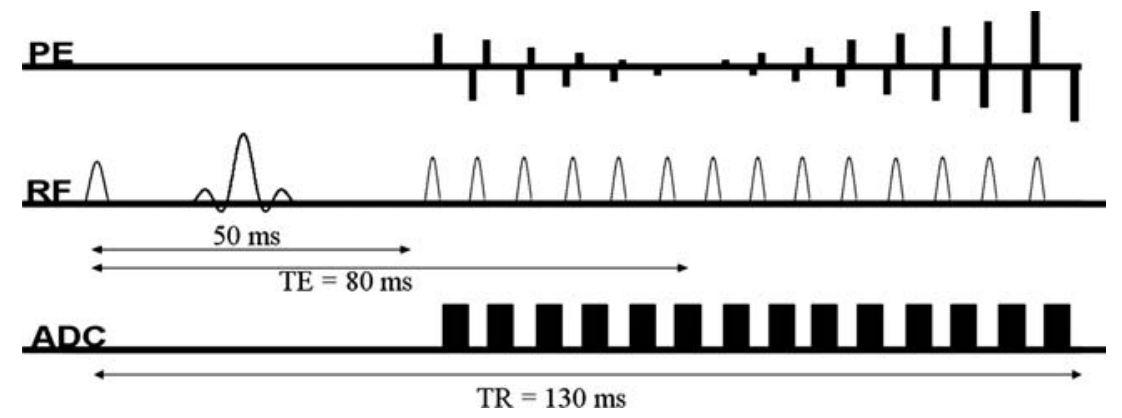

Fig. 1 Schematic of the HASTE sequence used for the activation studies. The initial $50 \mathrm{~ms}$ period sensitizes for BOLD contrast by allowing dynamic averaging. Using a combination of four-fold accelerated SENSE and 7/8 partial Fourier only $14 k$-space lines are sampled, reducing total slice acquisition time to $130 \mathrm{~ms}$, close to that of a typical SE-EPI experiment
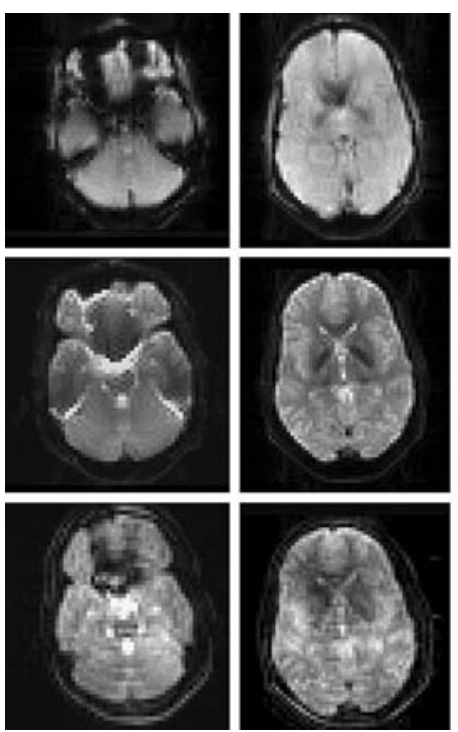
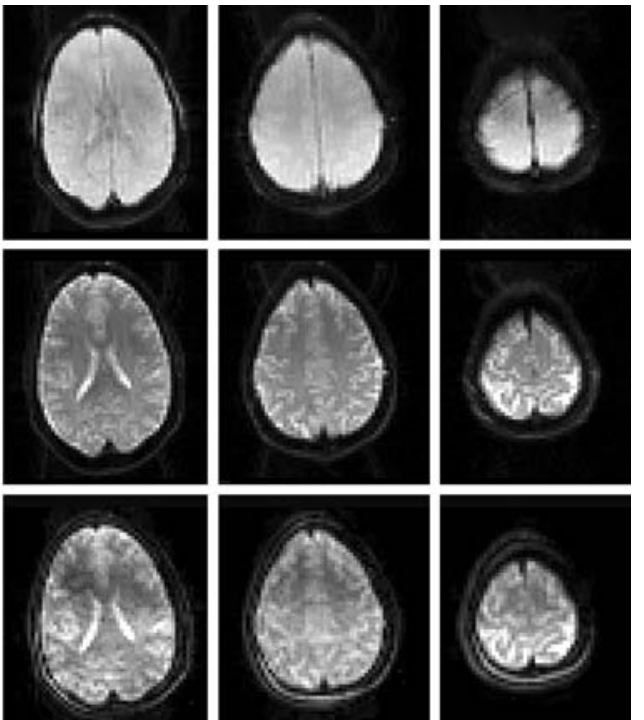

Fig. 2 Five brain slices spanning from inferior to superior brain, acquired with GE-EPI (top), SE-EPI (middle) and $\mathrm{T}_{2}$-prepared HASTE (bottom). The signal voids due to through-plane dephasing that are clearly present in the GE-EPI are absent in SE-EPI, but in-plane distortions remain and are particularly severe in the inferior brain. In both cases Nyquist ghosting can be observed. These EPI artifacts are not present in the HASTE images, how- ever the effect of coil sensitivity variations (e.g., in the left frontal regions) appears more pronounced. Traces of weak residual foldover that resembles the pattern of the $g$-map (see Fig. 3e) can be seen in the third and fourth slices. In no instance, however, did this result in a projection of artifactual visual activation into other brain areas rapidly growing number of coil elements [22] can yield much higher SNR: The $g$-noise penalty for acceleration factors much smaller than the number of coils would be much lower than for the $g$-factors observed here. Furthermore, $k$-space based reconstruction (e.g., GRAPPA or SMASH) would yield spatially almost invariant noise distributions, which might be regarded as favorable in an fMRI setup.

Magnetization transfer effects due to off-resonance power deposition posed no problem for the 5 -slice experiment in this study, but could potentially lead to signal attenuation and SNR reduction for short TR and/or longer refocusing pulse trains [23]. Substantial power reductions with only moderate SNR loss could be achieved by use of variable flip-angle schemes [24], and help improve fast multi-slice experiments.

A clear advantage of HASTE is the absence of inhomogeneity artifacts, yielding images that are free of both dropout and distortion. The latter allows precise mapping from functional to anatomical data, and can further be expected to be beneficial for group studies. It was shown in Ref. [25] that the application of post-hoc distortion correction to EPI data not only improves coregistration with the anatomical images, but also that group statistics on functional studies improved considerably. HASTE acquisitions should yield the same benefits, 

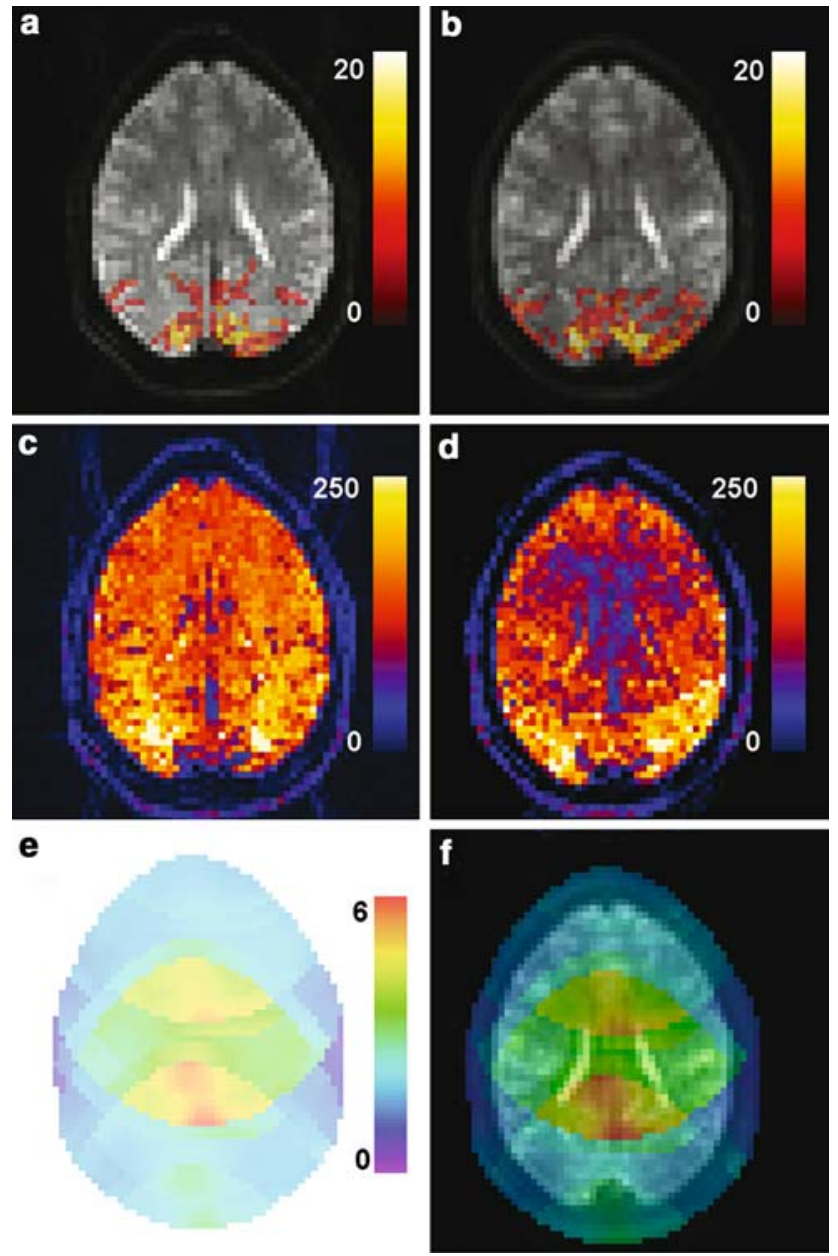

Fig. 3 Typical SE-EPI (a) and HASTE (b) images with activation overlays at $P<0.0001$ (color scale represents $t$-scores). Subfigures (c) and (d) show tSNR maps of the SE-EPI and HASTE data, respectively. Spatial variation in the latter resembles that of the $g$-map (e). The overlay of $g$-map on HASTE image (f) illustrates that visual areas are not strongly affected by $g$-noise

without the difficulties associated with correction methods that rely on the use of separately acquired reference data such as field maps.

In our implementation of the HASTE sequence, the signal acquisition is preceded by a preparation experiment for dynamic averaging that lasts $50 \mathrm{~ms}$. Ideally, the sequence would refocus the first echo at the optimal TE of $80 \mathrm{~ms}$ to maximize the dynamic averaging contribution, followed by centre-out acquisition of $k$-space, but at the price of a much longer total acquisition time. The compromise was chosen to achieve comparable slice acquisition times for SE-EPI and HASTE and permit a conservative comparison of the two methods. While we chose to use conventional SE-EPI without $k$-space undersampling for an unbiased assessment of relative functional sensitivities, SE-EPI readout times could also have been reduced by using factor $a$ parallel
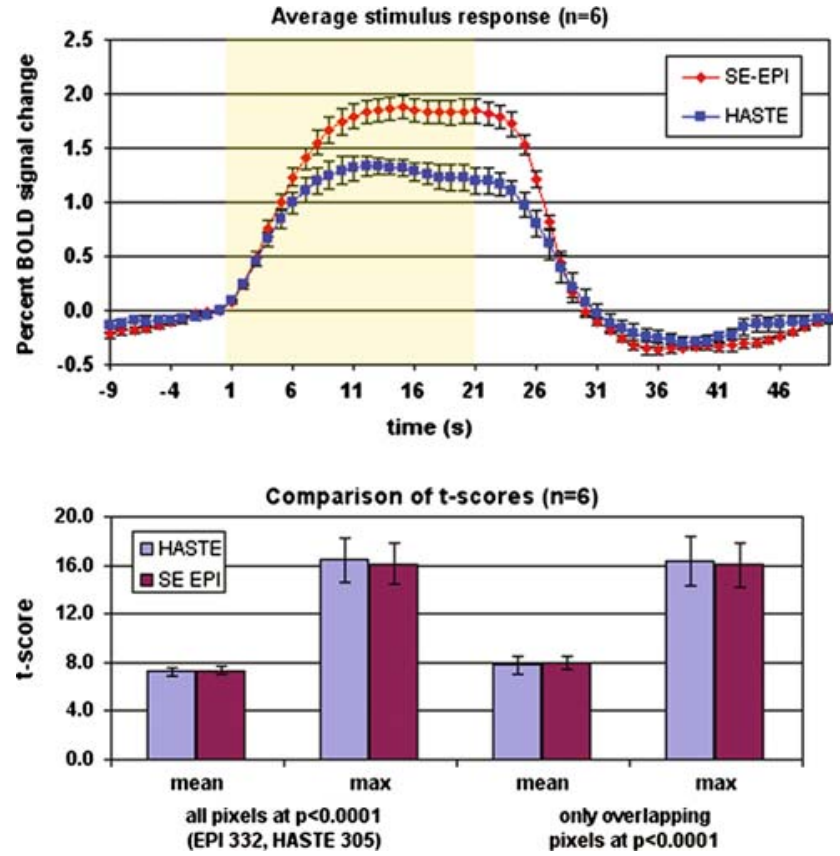

Fig. 4 Results of SE-EPI and HASTE BOLD measurements. Top Stimulus response curve considering all active pixels, averaged over subjects (error bars SEM over subjects, they reflect variation in signal change across subjects, but not sensitivity). Note that the average response curves of individual subjects were not normalized prior to averaging to allow comparison of the amplitude of the signal change between HASTE and EPI. The shaded region marks the $21 \mathrm{~s}$ stimulation period. Bottom Mean and maximum $t$-scores at $P<0.0001$ (subject average, error bars SD). For the calculation of overlapping pixels the distortion was assumed to be negligible

undersampling. This would have resulted in a $g \cdot \sqrt{ } a$ fold SNR penalty, and hence sensitivity reduction in favor of HASTE, but the benefit of $a$-fold reduction in EPI distortion $/ \mathrm{T}_{2} *$ blurring.

The presence of $\mathrm{T}_{2} *$ decay during the long readout train can be expected to contribute considerably to the functional signal changes in SE-EPI $[15,16]$. This most likely explains the majority of the observed difference between SE-EPI and HASTE. Phantom studies at $1.5 \mathrm{~T}$ have shown that up to $37 \%$ of the SE signal changes can arise due to static dephasing [26]. In vivo data at higher fields suggest an even larger contribution of $\mathrm{T}_{2}$ * effects, approximately $60 \%$ [27]. The amount of $\mathrm{T}_{2} *$ contribution depends on the spatial features of the activated areas, and would be largest for activation with higher spatial frequency components. Quantification of this effect is, hence, object dependent and beyond the scope of this paper.

A difference between SE-EPI and HASTE based $\mathrm{T}_{2}$ contrast is also likely to arise due to differences in refocusing interval: A standard SE-EPI protocol for fMRI will have a single refocusing pulse for TE $80 \mathrm{~ms}$, but our 
HASTE implementation has a spin echo preparation experiment with TE $50 \mathrm{~ms}$, followed by multiple refocusing pulses, with the $k$-space centre at TE $80 \mathrm{~ms}$. In partially oxygenated blood the proton exchange between erythrocytes and blood plasma, or diffusion in the local fields gradients arising from the presence of deoxyhemoglobin, results in a shortening of the effective intravascular $\mathrm{T}_{2}$ with increasing deoxyhemoglobin levels and increasing refocusing interval in CPMG sequences [28, 29]. This effect in itself forms the basis of the recently proposed VERVE fMRI contrast mechanism [30]. The rapid refocusing in the HASTE acquisitions thus implies elongation of the intravascular $\mathrm{T}_{2}$ compared to SE-EPI, where only a single refocusing pulse is applied, and hence a reduction in the functional sensitivity to intravascular signal changes. Similarly, increasing the number of refocusing pulses reduces the dynamic averaging contribution. Consequently both functional contrast mechanisms that contribute to spin-echo BOLD will be somewhat reduced in effect by the addition of extra refocusing pulses. It is difficult to assess the significance of this sensitivity loss, but as the difference in sensitivity between SE-EPI and HASTE in the present study is consistent with the literature regarding the $\mathrm{T}_{2} *$ sensitivity of SE-EPI $[26,27]$, it may be expected to be small.

A potential source of functional contrast is inflow effects. These could in principle explain some of the discrepancy in signal change if they contribute differently to the two methods. However, inflow should contribute little given the SE-EPI acquisition parameters [10], and be similar as the same TR of $2 \mathrm{~s}$ is used in both sequences.

An interesting question is raised by the fact that such a pronounced undershoot is observed in the SE data, particularly the HASTE. While it is generally accepted that the post-stimulus undershoot is in some way caused by the complex temporal relationship between the parameters cerebral blood volume (CBV), blood flow (CBF) and oxygen consumption $\left(\mathrm{CMRO}_{2}\right)$, there are two main types of explanation. First, the 'balloon model' family [31,32], which assumes a strong coupling between $\mathrm{CMRO}_{2}$ and $\mathrm{CBF}$, but a temporally varying relationship between of CBV and CBF. According to this model, following stimulation, delayed vascular compliance causes CBV to return to baseline more slowly than CBF and $\mathrm{CMRO}_{2}$, leading to a negative BOLD signal. The second type of explanation postulates a decoupling of CBF and $\mathrm{CMRO}_{2}$ : the undershoot is then caused by post-stimulus oxygen consumption that remains elevated even after CBF has returned to baseline [33].

We observed no difference between the normalized response curves of SE-EPI and HASTE, meaning that any $\mathrm{T}_{2} *$ effects present in the SE-EPI data equally affect both main BOLD signal and undershoot. These curves

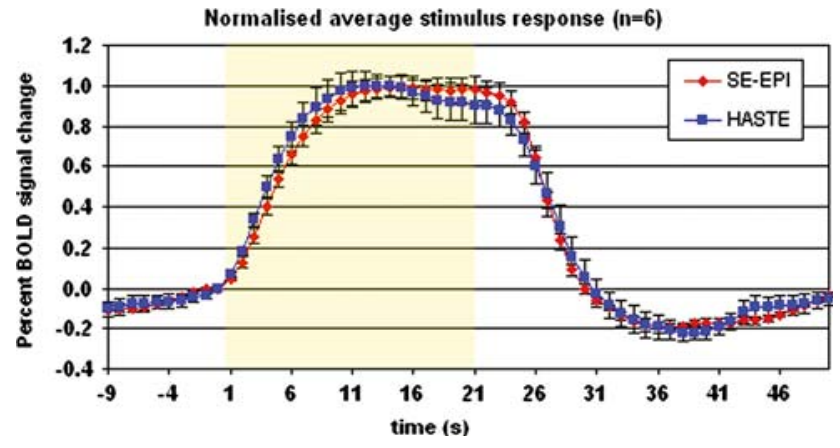

Fig. 5 Normalization of the curves shown in Fig. 4 results in both response curves being perfectly aligned, showing that HASTE sensitivity in the main and undershoot response scales by the same factor compared to SE-EPI

are shown in Fig. 5. Comparison of the normalized activation time courses with the GE-EPI data (not shown) which were simultaneously acquired with the SE-EPI also showed no difference in the undershoot. This suggests that the same combination of contrast mechanisms must be contributing to both the main BOLD signal and the post-stimulus undershoot. The Balloon model [31] predicts that the undershoot is largely due to extravascular static dephasing around the larger (downstream) vessels. An undershoot in the BOLD signal could, however, also be the result of intravascular $T_{2}$ changes as would result from sustained post-stimulus oxygen metabolism. Static dephasing effects are refocused by the SE experiment, so if the standard balloon model hypothesis were true, then the undershoot would have to be reduced in magnitude relative to the main response. Observation of the same signal contribution to both parts of the BOLD response thus rather excludes the interpretation of the undershoot as a downstream static dephasing effect, leaving changes in deoxyhemoglobin concentration as the more likely explanation. Such post-stimulus elevation in deoxyhemoglobin could be the result of ongoing oxygen metabolism after the return of CBV and CBF back to baseline, as suggested by Lu et al. [33]. That elevated CBV alone cannot be the cause for a poststimulus signal decrease has recently been reported by Yacoub et al. [34].

In conclusion, the application of fast spin echo sequences to fMRI was investigated. BOLD activation was robustly detected by HASTE acquisitions preceded by a preparation experiment for dynamic averaging. While the functional CNR in the occipital lobe was $\sim 30 \%$ lower than for a conventional SE-EPI acquisition of approximately equal TR, the absence of image artifacts makes HASTE a viable alternative where distortion free images are required. 
Acknowledgments The work presented in this paper was supported by STW (Dutch Science Foundation) grant NGT.6154. The authors thank Markus Barth for useful discussions, and Markus Klarhöfer for providing Matlab code on which the SENSE reconstruction was based.

\section{References}

1. Mansfield P (1984) Real-time echo-planar imaging by NMR. Br Med Bull 40(2):187-190

2. Ogawa S, Lee TM, Kay AR, Tank DW (1990) Brain magnetic resonance imaging with contrast dependent on blood oxygenation. Proc Natl Acad Sci USA 87(24):9868-9872

3. Williams DS, Detre JA, Leigh JS, Koretsky AP (1992) Magnetic resonance imaging of perfusion using spin inversion of arterial water. Proc Natl Acad Sci USA 89(1):212-216

4. Lu H, Golay X, Pekar JJ, van Zijl PCM (2003) Functional magnetic resonance Imaging based on changes in vascular space occupancy. Magn Reson Med 50(2):263-274

5. Duong TQ, Yacoub E, Adriany G, Hu X, Ugurbil K, Kim SG (2003) Microvascular BOLD contribution at 4 and $7 \mathrm{~T}$ in the human brain: gradient-echo and spin-echo fMRI with suppression of blood effects. Magn Reson Med 49(6):1019-1027

6. Parkes LM, Schwarzbach JV, Bouts AA, Deckers RH, Pullens P, Kerskens CM, Norris DG (2005) Quantifying the spatial resolution of the gradient echo and spin echo BOLD response at 3 Tesla. Magn Reson Med 54(6):1465-1472

7. Lee SP, Silva AC, Ugurbil K, Kim SG (1999) Diffusionweighted spin-echo fMRI at 9.4 T: microvascular/tissue contribution to BOLD signal changes. Magn Reson Med 42(5): 919-928

8. Yacoub E, Duong TQ, Van De Moortele PF, Lindquist M, Adriany G, Kim SG, Ugurbil K, Hu X (2003) Spin-echo fMRI in humans using high spatial resolutions and high magnetic fields. Magn Reson Med 49(4):655-664

9. Zhao F, Wang P, Kim SG (2004) Cortical depth-dependent gradient-echo and spin-echo BOLD fMRI at 9.4 T. Magn Reson Med 51(3):518-524

10. Yacoub E, Van De Moortele PF, Shmuel A, Ugurbil K (2005) Signal and noise characteristics of Hahn SE and GE BOLD fMRI at $7 \mathrm{~T}$ in humans. Neuroimage 24(3):738-750

11. Oja JM, Gillen J, Kauppinen RA, Kraut M, van Zijl PC (1999) Venous blood effects in spin-echo fMRI of human brain. Magn Reson Med 42(4):617-626

12. Norris DG, Zysset S, Mildner T, Wiggins CJ (2002) An investigation of the value of spin-echo-based fMRI using a Stroop color-word matching task and EPI at 3 T. Neuroimage 15(3):719-726

13. Jochimsen TH, Norris DG, Mildner T, Moller HE (2004) Quantifying the intra- and extravascular contributions to spinecho fMRI at 3 T. Magn Reson Med 52(4):724-732

14. Thulborn KR, Chang SY, Shen GX, Voyvodic JT (1997) Highresolution echo-planar fMRI of human visual cortex at 3.0 tesla. NMR Biomed 10(4-5):183-190

15. Duong TQ, Yacoub E, Adriany G, Hu X, Ugurbil K, Vaughan JT, Merkle H, Kim SG (2002) High-resolution, spin-echo BOLD, and CBF fMRI at 4 and 7T. Magn Reson Med 48(4):589-593

16. Birn RM, Bodurka JA, Petridou N, Bandettini PA (2004) Experimental determination of the effect of T2' changes in spin-echo EPI. In: Proceedings of the 12th annual meeting of ISMRM, Kyoto (Abstract 997)
17. Constable RT, Kennan RP, Puce A, McCarthy G, Gore JC (1994) Functional NMR imaging using fast spin echo at $1.5 \mathrm{~T}$. Magn Reson Med 31(6):686-690

18. Sodickson DK, Griswold MA, Jakob PM (1999) SMASH imaging. Magn Reson Imaging Clin N Am 7(2):237-254, vii-viii

19. Pruessmann KP, Weiger M, Scheidegger MB, Boesiger $P$ (1999) SENSE: sensitivity encoding for fast MRI. Magn Reson Med 42(5):952-962

20. Griswold MA, Jakob PM, Heidemann RM, Nittka M, Jellus V, Wang J, Kiefer B, Haase A (2002) Generalized autocalibrating partially parallel acquisitions (GRAPPA). Magn Reson Med 47(6):1202-1210

21. de Zwart JA, van Gelderen P, Kellman P, Duyn JH (2002) Application of sensitivity-encoded echo-planar imaging for blood oxygen level-dependent functional brain imaging. Magn Reson Med 48(6):1011-1020

22. Wiggins G, Potthast A, Triantafyllou C, Lin F, Benner T, Wiggins C, Wald L (2005) A 96-channel MRI-system with 23- and 90-channel phase array head coils at 1.5 T. In: Proceedings of the 13th annual meeting of ISMRM, Miami (Abstract 671)

23. Melki PS, Mulkern RV (1992) Magnetization transfer effects in multislice RARE sequences. Magn Reson Med 24(1):189195

24. Le Roux P, Hinks RS (1993) Stabilization of echo amplitudes in FSE sequences. Magn Reson Med 30(2):183-190

25. Cusack R, Brett M, Osswald K (2003) An evaluation of the use of magnetic field maps to undistort echo-planar images. NeuroImage 18(1):127-142

26. Birn RM, Bandettini PA (2002) The effect of T2' changes on spin-echo EPI-derived brain activation maps. In: Proceedings of the 10th annual meeting of ISMRM, Honululu (Abstract 1324)

27. Keilholz S, Silva A, Duyn J, Koretsky A (2005) The Contribution of $\mathrm{T}^{*}$ to Spin-echo EPI: implications for high-field fMRI studies. In: Proceedings of the 13th Annual Meeting of ISMRM, Miami (Abstract 32)

28. Bartha R, Michaeli S, Merkle H, Adriany G, Andersen P, Chen W, Ugurbil K, Garwood M (2002) In vivo 1H2O T2+ measurement in the human occipital lobe at $4 \mathrm{~T}$ and $7 \mathrm{~T}$ by Carr-Purcell MRI: detection of microscopic susceptibility contrast. Magn Reson Med 47(4):742-750

29. Stefanovic B, Sled JG, Pike GB (2003) Quantitative T2 in the occipital lobe: the role of the CPMG refocusing rate. J Magn Reson Imaging 18(3):302-309

30. Stefanovic B, Pike GB (2005) Venous refocusing for volume estimation: VERVE functional magnetic resonance imaging. Magn Reson Med 53(2):339-347

31. Buxton RB, Wong EC, Frank LR (1998) Dynamics of blood flow and oxygenation changes during brain activation: the balloon model. Magn Reson Med 39(6):855-864

32. Mandeville JB, Marota JJ, Ayata C, Zaharchuk G, Moskowitz MA, Rosen BR, Weisskoff RM (1999) Evidence of a cerebrovascular postarteriole windkessel with delayed compliance. J Cereb Blood Flow Metab 19(6):679-689

33. Lu HZ, Golay X, Pekar JJ, van Zijl PCM (2004) Sustained poststimulus elevation in cerebral oxygen utilization after vascular recovery. J Cerebr Blood F Met 24(7):764-770

34. Yacoub E, Ugurbil K, Harel N (2006) The spatial dependence of the poststimulus undershoot as revealed by high-resolution BOLD- and CBV-weighted fMRI. J Cereb Blood Flow Metab 26(5):634-644 